\title{
Desktop magnetic shielding system for the calibration of high-sensitivity magnetometers
}

\author{
Kunihisa Tashiro $^{1}$, Hiroyuki Wakiwaka ${ }^{1}$, Keishiro Matsumura ${ }^{1}$ and Keishi Okano ${ }^{1}$ \\ ${ }^{1}$ Spin device technology center, Shinshu University, Nagano, Wakasato 4-17-1, Japan
}

\begin{abstract}
The objective of the study is a development of desk-top magnetic shielding system for the calibration of high-sensitive magnetometers, and the evaluation of their ability to produce a uniform magnetic field. This system consists of hexagonal tubes and a cylinder. The hexagonal tubes have a double-layered structure, an opening and an advantage in axial shielding performance. The cylinder has openings and an advantage in transverse shielding performance. A solenoid-like coil is wound around the hexagonal tubes to produce a uniform magnetic field. From the experimental results, the magnetic shielding and the calibration field properties are demonstrated. Within the half length of the system, the achieved total magnetic field (dc component) is less than $200 \mathrm{nT}$ within $\pm 50 \mathrm{nT}$ deviation, and the measured shielding factor is larger than 250 . For producing a uniform magnetic field up to $10 \mathrm{kHz}$, we can use the $1 / 3$ length of the system.
\end{abstract}

Index Terms - Magnetic shield, shielding factor, calibration field, uniformity of magnetic field.

\section{INTRODUCTION}

Sensing of low-frequency magnetic fields is required in $N_{\text {many fields, and several kinds of magnetic sensors have }}$ already been proposed [1]. SQUID sensors are the most famous and can detect a weak magnetic field less than $1 \mathrm{pT}$. More than $90 \%$ of all SQUID systems sold and scientific papers published are related to neurological biomagnetic investigations [2]. Because these sensors use a superconducting interference device, the maintenance of liquid nitrogen/helium is necessary. Therefore, alternative magnetic sensors without low-temperature equipment have been also considered by many researchers [3]-[4].

For the calibrations of these high-sensitivity magnetometers, a coil system for generation of a uniform magnetic field and a magnetic shielding system with ferromagnetic material are known. A solenoid coil with infinite length has the best performance among coil systems. In practice, same-sized square coil systems have practical advantages in construction and usefulness. Most previous works proposed configurations which would zero as many high-order spatial derivatives of the magnetic field as possible at the center of the coil system [5]. Magnetic shielding systems are extremely expensive, heavy and have some practical problems. The BMSR-2 is well known as the best magnetically shielded room in the world [1]. It consists of seven mu-metal layers and one aluminum layer, whose total weight is 24.3 tons. Because the measured shielding factor (external / internal field) is extremely high, $10^{8}$ at $6 \mathrm{~Hz}$, we tend to expect that the internal dc magnetic field to be less than $500 \mathrm{fT}$. However, the reported value of the residual field is $2 \mathrm{nT}$ [6]. This fact points out the one of the practical problems. We should not so easily believe the value of the shielding factor, but rather also consider the other practical parameters.

For the reasons mentioned above, preparation of a magnetically shielded room is not realistic for researchers of

Manuscript received February 21, 2011. Corresponding author: Kunihisa Tashiro (e-mail: tashiro@ shinshu-u.ac.jp).

Digital Object Identifier inserted by IEEE high-sensitivity magnetometers. In order to meet demand, several designs of desk-top shielding system have been proposed [7]-[8]. Although a sphere is the best shape for a magnetically shielded enclosure, closed shape means no access inside. In practice, cylinders where the shape which has the theoretical expressions of the shielding factors are well known, and the open ends can be used for access inside. We can define two shielding factors, TSF (Transversal shielding factor) and $A S F$ (Axial shielding factor). In general, a cylindrical shield has an advantage in $T S F$, and multi-layered shells can achieve a better the value of TSF compared with a thick single shell. For a researcher of magnetic sensors, a magnetic circuit approach can help to understand this phenomenon [9].

We should consider the existence of a magnetic material which disturbs the uniformity of a field generated by a coil system. When a cylindrical magnetic shield is placed in a large coil system, the magnetic field inside the shield is not uniform. If the direction of the field is parallel to the center axis of the shield, the amplitude of the field from the ends to the center is exponentially decreased. This fact points out that the cylinder's $A S F$ from the ends to the center also changes exponentially [10]. It is well known that a Helmholtz coil consists of two circular coils of diameter $D$ with a distance of $0.5 \mathrm{D}$. If the coil is placed inside of a cylindrical shield, the distance should be $0.4716 D$ [11].

The objective of this study is the development of a desk-top magnetic shielding system for the calibration of highsensitivity magnetometers, and the evaluation of their ability to produce a uniform magnetic field. This system consists of hexagonal tubes and a cylinder. The hexagonal tubes have a double-layered structure, an opening and an advantage in axial shielding performance [12]. The cylinder has openings and an advantage in transverse shielding performance. A solenoidlike coil is wound around the hexagonal tubes to produce a uniform magnetic field.

\section{EXPERIMENT}

Fig. 1 shows our developed shielding system. It consists of 
the double-layered hexagonal tube and an outer cylinder. The outer length is $480 \mathrm{~mm}$, the outer diameter is $200 \mathrm{~mm}$, and the equivalent inner diameter is $130 \mathrm{~mm}$. A solenoid-like coil of 178 turns is wound around the outer surface of the tube for produce a uniform magnetic field along to the center axis. All magnetic layers were made of FINEMET sheet (MS-FR 47000M-TO, Hitachi Metals). Fig. 2 shows a schematic design of a building block in the tube. In order to connect the magnetic paths between the sides and bottom of the tube, one building block was composed of three U-shaped sheets. The easy axis direction of the sheets was parallel to the paths. The stacked number of building blocks is four for the inner, and twelve for the outer. The number of windings of the sheet in the cylinder is fifty-two and the easy axis direction of the sheet is circumferential.

The evaluation points of the developed system were the magnetic shielding and calibration field properties. Although the properties tended to be evaluated at the center position (one point) only, we evaluated the properties at many points along the center axis. For the magnetic shielding properties, we evaluated the residual magnetic flux density (dc components) of the geomagnetic field in our laboratory environment, and the shielding factors for a uniform field of $10 \mu \mathrm{T}_{\mathrm{p}-\mathrm{p}}$ in amplitude at $1 \mathrm{~Hz}$. For the calibration field, we generated a magnetic field of $100 \mathrm{nT}_{\mathrm{p}-\mathrm{p}}$ at the center point, and the frequency range was from $1 \mathrm{~Hz}$ to $10 \mathrm{kHz}$. The frequency responses of the magnetic field's uniformity and required current were measured.

Fig. 3 shows the configuration of the experimental setup for the measurement of the shielding factor. For the measurement, we used our developed Simple-Cubic-3 coil system (SC3) [13]. SC3 consists of three square coils connected in series, with a distance of half the side length of the coil. The side length is 2 $\mathrm{m}$, and the number of windings is 24:12:24. In the area within $500 \mathrm{~mm}$ radius from the center, SC3 can generate a uniform magnetic field within $\pm 1 \%$ deviation. The three sets of SC3 can generate uniform magnetic field in three-dimensions [14]. Because uniformity of the geomagnetic field was good in the wooden building, SC3 achieved the total magnetic field density less than $\pm 1 \mu \mathrm{T}$ area within $0.7 \mathrm{~m}$ in diameter [14]. Although the magnetic flux was disturbed by ferromagnetic object in our building, the uniformity in the vicinity of the center of SC3 was more or less all right. The achieved area was within $0.4 \mathrm{~m}$ in diameter.

In this paper, a three-axis fluxgate magnetometer (FVM400, MEDA Inc.) and an oscilloscope (TPS2014, Textronics) were used to observe the magnetic field, and a single-axis fluxgate magnetometer ( $\mu$ MAG-01, MEDA Inc.) and a FFT analyzer (CF-930, Ono Sokki) were used to measure the amplitude of magnetic field. For measurement in the $\mathrm{kHz}$ range, we used an induction magnetometer (3470, HIOKI).

\section{RESULTS}

In our laboratory environment, the total geomagnetic flux density measured at the center of SC3 was $24.9 \mu \mathrm{T}$. The $\mathrm{x}$ and $\mathrm{z}$ component were $25 \mu \mathrm{T}$, the y component was $-9 \mu \mathrm{T}$. We measured the residual magnetic flux density at the center point of the developed system. The center axis of the system was set to be parallel to the geomagnetic field of the horizontal component. The residual magnetic flux density of $\mathrm{x}$ component was $450 \mathrm{nT}$, the y component was $57 \mathrm{nT}$, and the $\mathrm{z}$ component was $120 \mathrm{nT}$. Although the shielding performance was not good in the $\mathrm{x}$ direction due to the existence of the opening, the total residual magnetic flux density was less than $500 \mathrm{nT}$.

Fig. 4 shows measured distributions of the residual magnetic flux density along the center axis. The center axis of the system was set to be parallel to the geomagnetic field of the horizontal component. A current passed through the solenoid-like coil was to be zero at the center of the magnetic field in the $\mathrm{x}$ component. The total residual magnetic field was less than $200 \mathrm{nT}$, and the area within $\pm 50 \mathrm{nT}$ was $240 \mathrm{~mm}$ along the axis. We also tested that the center axis of the system and the geomagnetic field of the horizontal component were perpendicular. The total magnetic flux density was less than $300 \mathrm{nT}$, and the area within $\pm 50 \mathrm{nT}$ was $180 \mathrm{~mm}$ along the axis.

Fig. 5 shows measured distributions of the shielding factor along the center axis. About the $\mathrm{y}$ and $\mathrm{z}$ components, the distributions of the shielding factor were same, and the value was changed from 250 at the center to 700 at the bottom. About the $\mathrm{x}$ component, the profile of the distribution was similar as other components, and the value changed from 40 at the center to 70 at the bottom. We also measured the frequency response of the shielding factor at the center. It was confirmed that measured values of the shielding factor did not change when the frequency was less than $1 \mathrm{kHz}$.

Fig. 6 shows the measured magnetic field distribution in the $x$ direction on the center axis of the system, as a parameter of the frequency. In order to generate the $\mathrm{x}$ component of the field $100 \mathrm{nT}_{\mathrm{p}-\mathrm{p}}$, a current to be fed to the solenoid-like coil was controlled. From the results, the current-to-field ratio of the coil was a constant value of $70.18 \mathrm{nT} / \mathrm{mA}$ when the frequency was less than $500 \mathrm{~Hz}$. If the frequency was larger than $1 \mathrm{kHz}$, the ratio was decreased. For example, the ratio was $1 / 4$ times at $10 \mathrm{kHz}$. The area of the uniform magnetic field within $\pm 3 \%$ deviation was $150 \mathrm{~mm}$ along the center axis. While the area was small if the frequency was high, the area within $\pm 10 \%$ deviation was $200 \mathrm{~mm}$ when the frequency was $10 \mathrm{kHz}$.

\section{Discussion}

It is not easy to design a sufficient value for $A S F$. In other words, the value is limited by the length to diameter ratio $L / D$ even if the magnetic material has infinite permeability. For example, the values at $L / D=3$ are 100,000 for $T S F$ and 300 for $A S F$. In order to improve the situation, well-designed end caps [7] or magnetic shaking techniques [8] have been proposed. Although PC permalloy is most famous as a shielding material, its magnetic properties degrade from induced stress. Also, the imperfect connection between the cylinder and end-caps lead to leakage of magnetic flux inside. With the magnetic shaking technique, an amorphous ribbon having a rectangular B-H loop exhibits a large incremental permeability of 500,000. However, the required amplitude of 
the shaking field is proportional to the shaking frequency, and the leakage in the shield is not negligible. Because the typical leakage field is a few $\mu \mathrm{T}$, additional passive shells should be installed to reduce it.

From the experimental results, we successfully demonstrated the magnetic shielding and calibration field properties of the system and showed the specifications to discuss its practical use. Table 1 shows the specifications. In our laboratory environment, the total residual magnetic field (dc magnetic field) was less than $200 \mathrm{nT}$ within $\pm 50 \mathrm{nT}$ deviation in the half length of the system. A cylindrical magnetic shield of similar size was presented in ref [7], which achieved the density ten times smaller than our results. The values were less than $20 \mathrm{nT}$ in the axial direction, $5 \mathrm{nT}$ in the radial direction. Compared with conventional cylindrical shields, our system could not equipped a toroidally wound coil which can be used for degaussing the magnetic layers. In contrast, our developed system can control the density in the axial direction (x direction). If we adjust the density at the +12 $\mathrm{cm}$ from the center, the area of the density less than $10 \mathrm{nT}$ is relatively wide as $6 \mathrm{~cm}$. In this area, the deviation of the density in radial direction ( $\mathrm{y}$ and $\mathrm{z}$ direction) is also small. From the results with a uniform magnetic field of $1 \mathrm{~Hz}$ at 10 $\mu \mathrm{T}$, the measured shielding factor was larger than 250 , which was measured within the half length of the system. At the center of SC3, typical magnetic field from power-line was a few nT. We conclude that the shielding factor is sufficient because the corresponding amplitude is less than 100 pT. Feeding a current to the solenoid-like coil with frequencies between from $1 \mathrm{~Hz}$ to $10 \mathrm{kHz}$, the system produced a uniform magnetic field of $100 \mathrm{nT}_{\mathrm{p}-\mathrm{p}}$ within $\pm 10 \%$ deviation of $1 / 3$ of the length of the system. Because of the uniformity, the calibration field could be also used for active compensation for the axial shielding performance.

\section{CONCLUSION}

We presented a desktop magnetic shielding system for the calibration of high-sensitivity magnetometers. In order to evaluate the system for practical use, the uniformity of the magnetic field inside the system was focused on. Our measured properties were the residual magnetic field, the shielding factor, and the calibration field. From the experimental results, we successfully demonstrated the magnetic shielding and calibration field properties of the system and showed the specifications for discussing its practical use.

\section{REFERENCES}

[1] J. Lenz and A. S. Edelstein, "Magnetic sensors and their applications," IEEE Sensors Journal, vol. 6, pp. 631-649, 2006.

[2] H. Koch, "SQUID Magnetocardiography: Status and Perspectives," IEEE Trans. Appl. Super., vol. 11, pp. 49-59, 2001.

[3] D. Robbes, C. Dolabdjian, S. Saez, Y. Monfort, G. Kaiser, and P. Ciureanu, "Highly sensitive uncooled magnetometers: State of the art. Superconducting magnetic hybrid magnetometers, an alternative to SQUIDs?," IEEE Trans. Appl. Super., vol. 11, pp. 629-634, 2001.

[4] D. J. Mapps, "Remote magnetic sensing of people," Sensors and Actuators A, vol. 106, pp. 321-325, 2003.
[5] J. L. Kirschvink, "Uniform magnetic fields and double-wrapped coil system," Bioelectromagnetics, vol. 13, pp. 401-411, 1992.

[6] F. Thiel, A. Schnabel, S. Knappe-Gruneberg, D. Stollfuss, and M. Burghoff, "Proposal of a demagnetization function," IEEE Trans. on Magn., vol. 43, pp. 2959-2961, 2007.

[7] E. A. Burt and C. R. Ekstrom, "Optimal three-layer cylindrical magnetic shield sets for scientific applications," Rev. Sci. Instrim., vol. 73, pp. 2699-2704, 2002.

[8] K. Nagashima, I. Sasada, and K. Tashiro, "High-performance bench-top cylindrical magnetic shield with magnetic shaking enhancement," IEEE Trans. on Magn., vol. 38, pp. 3335-3337, Sep 2002.

[9] E. Paperno and I. Sasada, "Magnetic circuit approach to Magnetic shielding," J. Magn. Soc. Japan, vol. 24, 2000.

[10] K. Nagashima, I. Sasada, and K. Tashiro, "Adaptive compensation of magnetic fields inside an open cylindrical magnetic shield," IEEE Trans. on Magn., vol. 39, pp. 3223-3225, Sep 2003.

[11] M. Hosoya and E. Goto, "Coils for generating uniform fields in a cylindrical ferromagnetic shield," Rev. Sci. Instrim., vol. 62, pp. 2472$2475,1991$.

[12] A. Matsuoka, K. Matsumura, A. Kubota, K. Tashiro and H. Wakiwaka, " A study on magnetic shielding enclosure with openings with coil systems", Journal of the Japan Society of Applied Electromagnetics and Mechanics, Vol. 18, No. 3, pp. 215-220, 2010. (in Japanese)

[13] K. Tashiro, A. Matsuoka, and H. Wakiwaka, "Simple-Box-9 coil system: A novel approach to design of a square coil system for producing uniform magnetic fields," Materials Science Forum, vol. 670, pp. 275-283, 2011.

[14] A. Matsuoka, K. Matsumura, A. Kubota, K. Tashiro, and H. Wakiwaka, "Residual magnetization measurements of a motor to be used in satellites," Proc. of SPIE, vol. 7500, pp. 750012-1 - 750012-6, 2009.

TABLE 1

SPECIFICATIONS OF THE SYSTEM

\begin{tabular}{|c|c|}
\hline Property & Values \\
\hline Outer length & $480 \mathrm{~mm}$ \\
\hline Outer diameter & $200 \mathrm{~mm}$ \\
\hline Inner diameter (equivalent) & $130 \mathrm{~mm}$ \\
\hline \multicolumn{2}{|l|}{ Total residual magnetic field } \\
\hline At the center & $\sim 200 \mathrm{nT}$ \\
\hline Area of uniformity within $\pm 50 \mathrm{nT}$ & $240 \mathrm{~mm}$ \\
\hline \multicolumn{2}{|l|}{ Magnetic shielding factor } \\
\hline$T S F$ (y or z component) & 250(center), 700(bottom) \\
\hline$A S F$ ( $\mathrm{x}$ component $)$ & 40(center), 70(bottom) \\
\hline \multicolumn{2}{|l|}{ Calibration field } \\
\hline Current-to-field ratio & $70.18 \mathrm{nT} / \mathrm{mA}$ (up to $500 \mathrm{~Hz}$ ) \\
\hline Area of uniformity within $\pm 3 \%$ & $150 \mathrm{~mm}$ \\
\hline within $\pm 10 \%$ & $200 \mathrm{~mm}$ \\
\hline
\end{tabular}




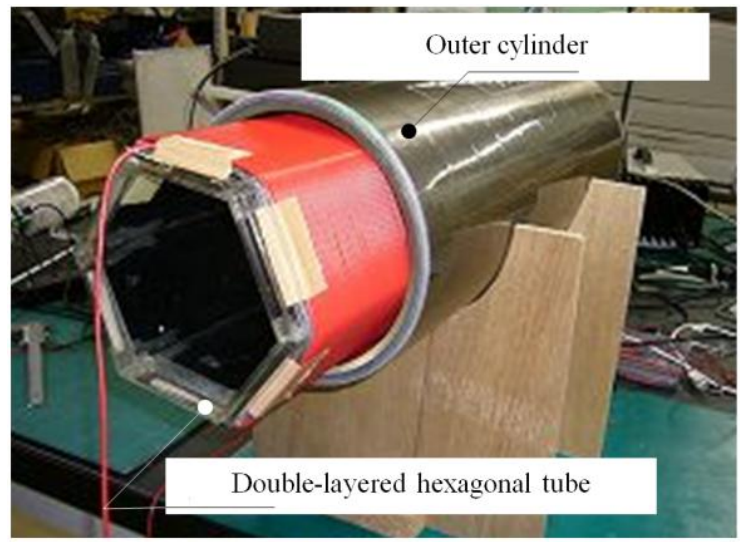

Fig. 1. Our developed desktop magnetic shielding system. It consisted of a double-layered hexagonal tube and an outer cylinder. A solenoid-like coil of 178 turns was wound around the outer surface.

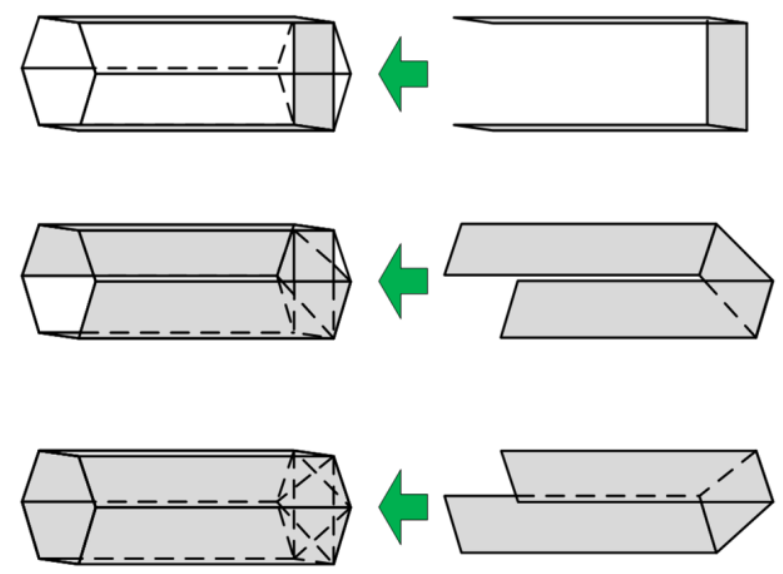

Fig. 2. A schematic design showing the building block in the hexagonal tubes. One building block is composed of three U-shaped sheets. The stacked number of building blocks was four for the inner, and twelve for the outer.

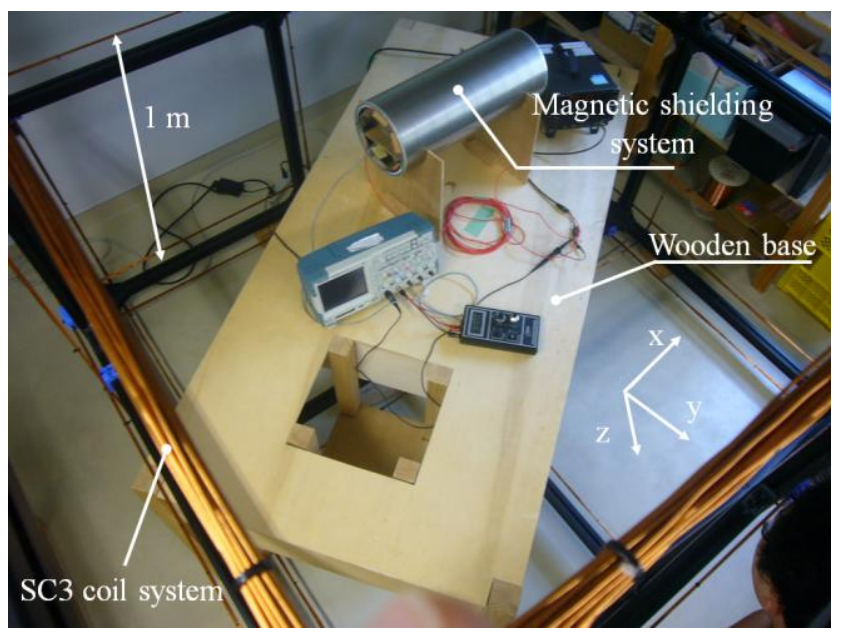

Fig. 3. Configuration of the experimental setup for the measurement of the shielding factor. The definition of the axes is also shown in the figure.

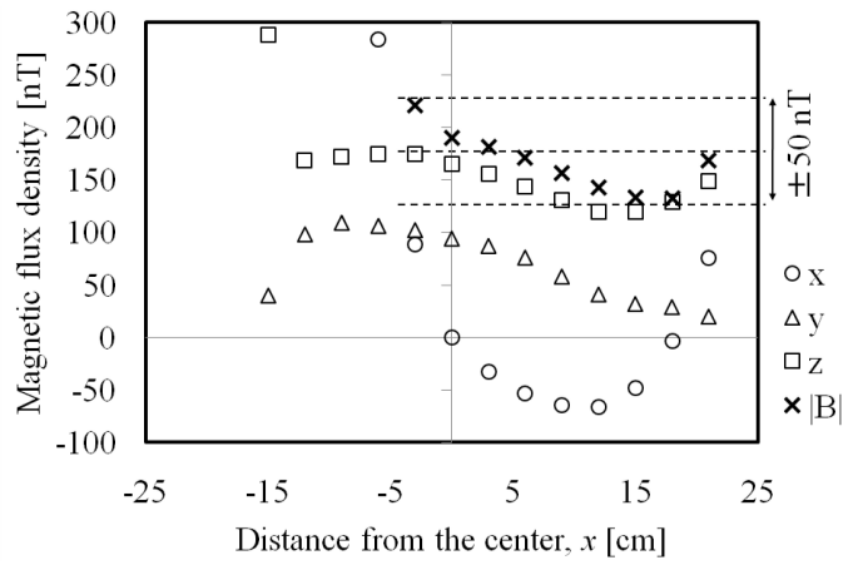

Fig. 4. Measured distributions of the residual magnetic flux density along the center axis. The center axis of the system was set to be parallel to the geomagnetic field of the horizontal component. A current passed through the solenoid-like coil was set to be zero at the center of the magnetic field in the $\mathrm{x}$ component.

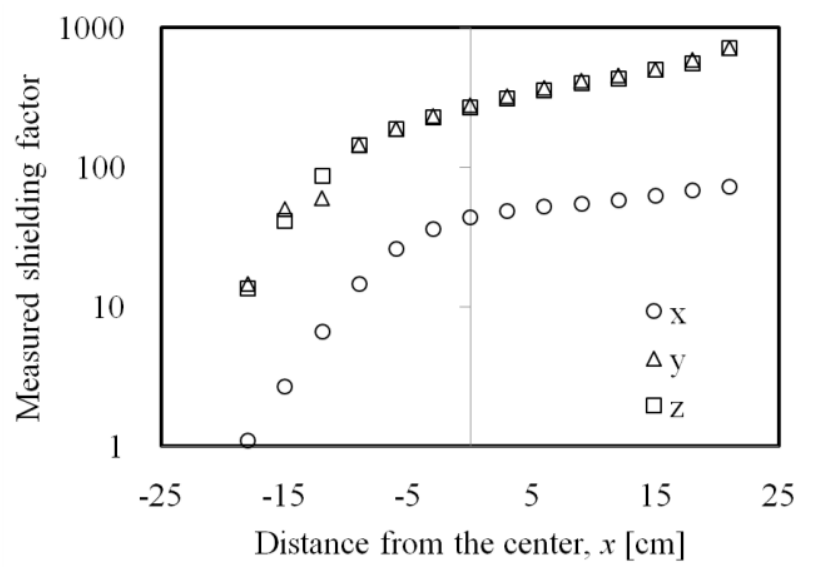

Fig. 5. Measured distributions of the shielding factor along the center axis. The condition of the uniform magnetic field was same as Fig. 4.

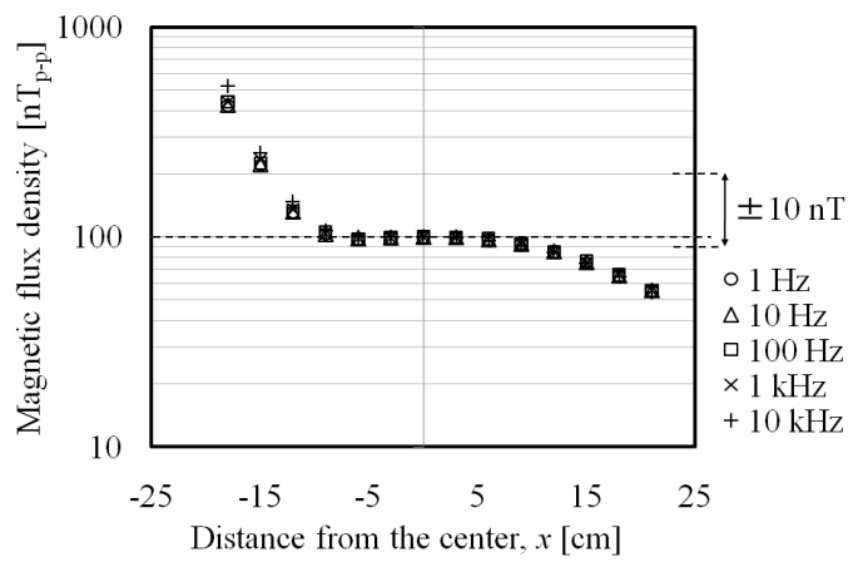

Fig. 6. Measured magnetic field distribution in the $\mathrm{x}$ direction on the center axis of the system, as a parameter of the frequency. In order to generate the $\mathrm{x}$ component of the field $100 \mathrm{nTp}$-p, a current to be fed to the solenoid-like coil was controlled. 